\title{
Influence of postpartum supplementation with vitamin A on the levels of immunoglobulin $A$ in human colostrum
}

\author{
Mayara S. R. Lima, ${ }^{1}$ Penha P. C. Ribeiro, ${ }^{1}$ Jovilma M. S. Medeiros, ${ }^{1}$ \\ Isabelle F. Silva, ${ }^{1}$ Ana C. P. Medeiros, ${ }^{2}$ Roberto Dimenstein ${ }^{3}$
}

\begin{abstract}
Objective: To evaluate the influence of supplementation with retinyl palmitate in the immediate postpartum period on the levels of secretory immunoglobulin A (SIgA) in colostrum.

Methods: A clinical trial was conducted among 96 recently-delivered mothers treated at a public maternity hospital, divided into control group, which was not supplemented $(n=44)$, and test group, supplemented on the first day postpartum $(n=52$ ). Samples of $2 \mathrm{~mL}$ of colostrum were collected on the first 2 days postpartum. SIgA was measured by turbidimetry and data were analyzed by the Student $t$ test.

Results: Before supplementation, the average SIgA was $829.1 \pm 337.6 \mathrm{mg} / \mathrm{dL}$ in the control group and $827.3 \pm 249.8 \mathrm{mg} / \mathrm{dL}$ in the test group $(p=0.52)$. After supplementation, the average SIgA was $343.9 \pm 177.2$ $\mathrm{mg} / \mathrm{dL}$ in the unsupplemented group and $501.2 \pm 54.5 \mathrm{mg} / \mathrm{dL}$ in the supplemented group $(p<0.00001)$.

Conclusion: The colostrum of women supplemented with retinyl palmitate has higher levels of SIgA, which suggests that the production of antibodies is modulated by vitamin A.
\end{abstract}

J Pediatr (Rio J). 2012;88(2):115-8: Vitamin A, secretory immunoglobulin A, colostrum, dietary supplements, clinical trial.

\section{Introduction}

Breast milk is the best form of nutrition for the newborn (NB), due to its ideal nutritional characteristics and its immunological and psychological advantages that are important to the reduction of infant morbidity and mortality. ${ }^{1}$

Human colostrum, the milk secretion produced in the first days postpartum, is rich in antibodies, providing immunological benefits to the NB, and thus can be compared with a vaccine. ${ }^{2}$ The main antibody in human milk is secretory immunoglobulin $A$ (SIgA), which is produced by the secretory cells of the breast and released by enteromammary circulation, protecting the NB against microbial antigens and allergens. ${ }^{3}$

Besides having a higher concentration of immunological components, colostrum is rich in fat soluble vitamins like vitamin A or retinol, also known as anti-infective vitamin, due to its importance to immune function. ${ }^{4}$

Nursing mothers and breastfed children are among the high-risk groups for vitamin A deficiency (VAD), and such deficiency in the mother can lead to an inadequate

1. Acadêmica de Nutrição, Centro de Ciências da Saúde, Departamento de Nutrição, Universidade Federal do Rio Grande do Norte (UFRN), Natal, RN, Brazil. 2. Mestre. Bioquímica, Centro de Biociências, Departamento de Bioquímica, UFRN, Natal, RN, Brazil.

3. Doutor. Professor, Centro de Biociências, Departamento de Bioquímica, Laboratório de Bioquímica de Alimentos e Nutrição, UFRN, Natal, RN, Brazil.

No conflicts of interest declared concerning the publication of this article.

Financial support: Coordenação de Aperfeiçoamento de Pessoal de Nível Superior (CAPES).

Suggested citation: Lima MS, Ribeiro PP, Medeiros JM, Silva IF, Medeiros AC, Dimenstein R. Influence of postpartum supplementation with vitamin A on the levels of immunoglobulin A in human colostrum. J Pediatr (Rio J). 2012;88(2):115-8.

Manuscript submitted Jul 2 2011, accepted for publication Nov 162011.

http://dx.doi.org/10.2223/JPED.2162 
vitamin A status in the NB, which increases the risk of infant morbidity and mortality. ${ }^{5}$ Thus, vitamin A supplementation is a useful public health strategy to improve child survival by decreasing the risk of morbidity of infectious origin from diseases such as measles, severe diarrhea, HIV, and possibly malaria and intestinal helminthiases. ${ }^{6}$

Nikawa et al. ${ }^{7}$ tested the influence of supplementation with retinyl acetate on the production of immunoglobulin A $(\operatorname{IgA})$ in the mucosa of mice and observed that, in most animals, there was an increase in IgA concentration after vitamin A supplementation.

Other studies in mice investigated the influence of supplementation with beta-carotene (pro-vitamin A) on IgA levels in milk and concluded that supplementation with this substance during pregnancy and lactation is useful for enhancing IgA transfer to maternal milk. ${ }^{8}$

Considering the importance of vitamin $A$ to immune function and the evidence of an increase in IgA production after vitamin A supplementation in animals, the present study aimed at evaluating the influence of supplementation with retinyl palmitate in the immediate postpartum period on SIgA levels in the colostrums of recently-delivered mothers treated at a public maternity hospital.

\section{Methods}

This was a clinical trial that used a convenience sample consisting of volunteer recently-delivered mothers treated at a maternity teaching hospital (Maternidade Escola Januário Cicco, Natal, Brazil).

The study protocol was approved by the Research Ethics Committee of Hospital Universitário Onofre Lopes, affiliated with Universidade Federal do Rio Grande do Norte (UFRN), (protocol n० 284/09) and by the board of directors of Maternidade Escola Januário Cicco.

The sample size was calculated inferentially, using means and dispersion measurements from a previous study. ${ }^{9}$ The standard deviation of maternal retinol levels in the first month postpartum was assumed to be not greater than $14.9 \mu \mathrm{g} / \mathrm{dL}$. Consequently, it would be necessary to recruit at least 42 women in each group, in order to detect a difference of $10.0 \mu \mathrm{g} / \mathrm{dL}$, with $80 \%$ power, $95 \%$ confidence interval, and $25 \%$ loss to follow-up.

Inclusion criteria for the study were: women who gave birth to a singleton full-term or pre-term infant with no malformations. Women with diseases such as diabetes, neoplasias, gastrointestinal and liver disorders, cardiopathies, infectious diseases, syphilis, and HIV or who reported use of vitamin A supplements during pregnancy were not included in the study. The sample comprised 96 recently-delivered mothers, 44 of which were in the control group (unsupplemented) and 52 in the supplemented group (supplemented with a megadose of 200,000 IU of retinyl palmitate in the immediate postpartum period).
Data collection was divided into two stages, which took place in different periods. At first, the control group was formed, consisting of healthy women admitted to the maternity hospital during the data collection period who met the inclusion criteria and agreed to participate in the study. This was followed by data collection from the test group, using the same procedures and criteria above mentioned, except by the supplementation given to the mothers in the test group.

After the purposes of the study had been explained and mothers had given their free and informed consent, a 2-mL colostrums sample was collected after overnight fasting, always in the morning. Next, a capsule of 200,000 IU (60 $\mathrm{mg}$ ) of retinyl palmitate was administered to the mothers in the test group, while the mothers in the control group did not receive such supplementation. On the following day, after 24 hours, colostrum was collected again before breakfast. After the second collection, women in the control group also received supplementation with retinyl palmitate, as recommended by the Brazilian Ministry of Health. Colostrum was obtained by manual expression of a single breast at the beginning and at the end of the breastfeed in order to avoid variation in fat content. These procedures were carried out by undergraduate research students who were trained for that purpose.

Colostrum samples were collected in a polypropylene tube protected from the sunlight and taken under refrigeration to the Laboratory of Food Biochemistry and Nutrition from the Department of Biochemistry at the Biosciences Center of UFRN, and stored at $-20^{\circ} \mathrm{C}$ until analysis. Maternal and infant data, as well as information on delivery, were obtained from the medical records of each mother.

SIgA was evaluated by turbidimetry, using a kit for measuring IgA manufactured by In Vitro Diagnóstica (Itabira, Brazil). This method is based on the reaction between immunoglobulins - as antigen - and the specific antibody - as antibody. Such reaction usually forms insoluble immunoprecipitates, involving thus turbidity, which is measured by a spectrophotometer

It is known that SIgA is concentrated in the whey, although a small amount is limited to milk fat globules ${ }^{10}$; therefore, milk was previously centrifuged (500 x g) for 10 minutes, and the solid and fatty layer was discarded, while the whey was collected and refrigerated at $-20{ }^{\circ} \mathrm{C}$. In order to perform the test, whey was diluted with $0.9 \%$ saline $\mathrm{NaCl}$ solution in the proportion of $1: 20$; accordingly, for each $50 \mu \mathrm{L}$ of whey, $1,000 \mu \mathrm{L}$ of solution were added. A total of $20 \mu \mathrm{L}$ were taken from the diluted whey and added to $1,000 \mu \mathrm{L}$ of IgA antiserum (AS-A), which was previously prepared for use and contained ethylene glycol as accelerator. The standard solution underwent the same procedure. Then, tubes and samples were carefully homogenized, along with standard and blank solutions; they were incubated in a water bath at $37^{\circ} \mathrm{C}$ for 10 
minutes, so as to allow the occurrence of the reaction between SIgA and its corresponding antiserum. The turbidity generated by the reaction was detected at 340 $\mathrm{nm}$ by a spectrophotometer (Femto, São Paulo, Brazil). The immunoglobulin concentration was calculated using IgA pattern (IgA Cat. 11.002), and values are given in $\mathrm{mg} / \mathrm{dL}$.

IgA levels were expressed as mean and standard deviation. The Student $t$ test was used to test the differences between the means of parametric numerical data, and differences were significant when $p<0.05$.

\section{Results}

On the first day postpartum $(0 \mathrm{~h})$, the average concentration of SIgA in the colostrums of the mothers in the control group was $829.1 \pm 337.6 \mathrm{mg} / \mathrm{dL}$, similar to that of those in the test group $(827.3 \pm 249.8 \mathrm{mg} / \mathrm{dL})$, since the women in the latter group had not received supplementation yet. Therefore, there was no significant difference between mean immunoglobulin levels of both groups on the first day postpartum $(p=0.52)$.

On the following day, 24 hours after receiving a 200,000 IU dose of retinyl palmitate, women in the supplemented group showed higher SIgA levels in their colostrum compared with mothers who were not supplemented $(501.2 \pm 54.5 \mathrm{mg} / \mathrm{dL}$ and $343.9 \pm 177.2$ $\mathrm{mg} / \mathrm{dL}$ respectively); therefore, there was a statistically significant difference between the means of the groups $(p<0.00001)$ (Figure 1).

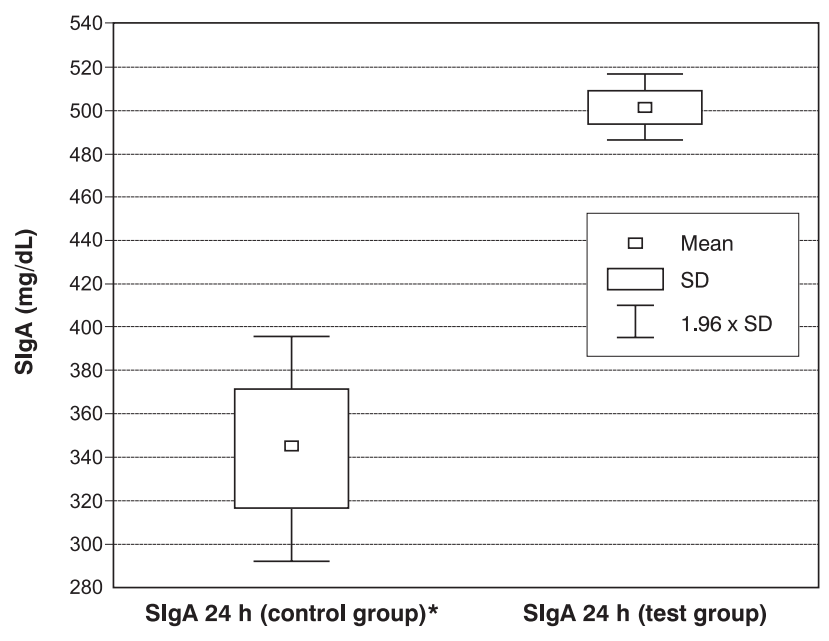

$\mathrm{SD}=$ standard deviation; SIgA = secretory immunoglobulin A.

* Statiscally significant difference in comparison with the test group Student $t$ test for independent samples $(p<0.0001)$.

Figure 1 - SigA levels $(\mathrm{mg} / \mathrm{dL})$ in the colostrum of mothers in control and test groups on the second day postpartum (24 hours after the test group received supplementation)
It was observed that both groups showed lower SIgA levels in colostrum 24 hours after delivery, as compared with those found on the first day. However, the decrease in immunoglobulin levels in the unsupplemented group was $58.4 \%$, while it reached $39.5 \%$ in the vitamin A-supplemented group.

\section{Discussion}

The fact that mothers supplemented with retinyl palmitate had higher SIgA levels has demonstrated the importance of vitamin $A$ in immunity and has reinforced the hypothesis that this vitamin is related to higher SIgA levels in the milk of breastfeeding mothers.

According to Semba, ${ }^{11}$ vitamin A supplementation has been shown to reduce the incidence and the severity of several diseases. Experiences with animals suggest that vitamin $A$ and related retinoids modulate many elements of the immune system, including keratin and mucin expressions, lymphopoiesis, apoptosis, cytokine production, neutrophyl function, natural killer cells, monocytes and macrophages, $\mathrm{T}$ and $\mathrm{B}$ lymphocytes and immunoglobulin production. ${ }^{12}$

An indirect measure of the potential effect of vitamin A supplementation on B cell function is the production of antibodies. This indirect effect, according to Duriancik et al., ${ }^{12}$ probably occurs due to the influence of the vitamin on antigen-presenting cells, which have the function of presenting the antigen to B lymphocytes, favoring immunoglobulin synthesis.

Moreover, a study in mice showed that the nuclear factor of activated T cells is lower in VAD and higher in the presence of vitamin $A$, and that this factor is required to enable IgA synthesis by $B$ cells. Therefore, according to the authors, vitamin A plays a critical role in immune response, because its intake induces IgA production by increasing the concentration of the factor that stimulates the cells which produces this immunoglobulin. ${ }^{13}$

The mechanisms that lead to the higher presence of SIgA in human milk after vitamin A supplementation are not totally elucidated yet; thus, further studies on the subject are needed, even to investigate which factors are involved in such a fast response to supplementation as that observed in our study.

Additionally, a limitation of this study was that it did not examine SIgA levels in colostrum during a longer period of time after supplementation, which could make it possible to analyze immunoglobulin content in mature milk as well, in order to investigate if maternal supplementation shows long-term benefits on SIgA content in milk.

It was observed that SIgA concentration in women's colostrum on the first day postpartum was nearly twice as high as the concentration of this immunoglobulin in 
colostrum on the second day. However, this decrease was expected, since, according to Ballabio et al., ${ }^{14}$ SIgA concentration in colostrum significantly decreases in the first days postpartum. However, the total amount of this immunoglobulin ingested by the child remains practically unchanged during the first 2 to 3 months of life, due to an increase in infant milk consumption. ${ }^{15}$

Although there is a decrease in immunoglobulin levels, it was observed that mothers supplemented with retinyl palmitate in the immediate postpartum period showed, on average, $30 \%$ more SIgA in colostrum on the second day postpartum when compared with unsupplemented mothers.

Thus, the results obtained show that vitamin $A$ is important in immunity, because they suggest that the production of antibodies is modulated by this vitamin, which indicates that supplementation may be considered an effective alternative to improve the immunity and nutritional status of nursing women and their NBs.

\section{Acknowledgements}

We would like to thank Maternidade Escola Januário Cicco and the mothers who participated in the study for allowing data collection.

\section{References}

1. Marques RF, Lopez FA, Braga JA. O crescimento de crianças alimentadas com leite materno exclusivo nos primeiros 6 meses de vida. J Pediatr (Rio J). 2004;80:99-105.

2. Brasil. Ministério da Saúde. Secretaria de Atenção à Saúde. Promovendo o aleitamento materno. $2^{\mathrm{a}}$ ed. Brasília: Ministério da Saúde; 2007. Álbum seriado.

3. Fagarasan S, Kawamoto S, Kanagawa O, Suzuki K. Adaptive immune regulation in the gut: $T$ cell-dependent and $T$ cell-independent IgA synthesis. Annu Rev Immunol. 2010;28:243-73.

4. Stephensen CB. Vitamin $A$, infection, and immune function. Annu Rev Nutr. 2001;21:167-92.
5. Ramalho RA, Flores H, Accioly E, Saunders C. Associação entre deficiência de vitamina A e situação socioeconômica de mães e recém-nascidos. Rev Assoc Med Bras. 2006;52:170-5.

6. Villamor E, Fawzi WW. Effects of vitamin A supplementation on immune responses and correlation with clinical outcomes. Clin Microbiol Rev. 2005; 18:446-64.

7. Nikawa T, Ikemoto M, Kano M, Tokuoka K, Hirasaka K, Uehara $\mathrm{S}$, et al. Impaired vitamin A-mediated mucosal IgA response in IL-5 receptor-knockout mice. Biochem Biophys Res Commun. 2001;285:546-9.

8. Nishiyama Y, Sugimoto M, Ikeda S, Kume S. Supplemental B-carotene increases IgA-secreting cells in mammary gland and IgA transfer from milk to neonatal mice. $\mathrm{Br}$ J Nutr. 2011;105:24-30.

9. Ayah RA, Mwaniki DL, Magnussen P, Tedstone AE, Marshall T, Alusala D, et al. The effects of maternal and infant vitamin A supplementation on vitamin A status: a randomised trial in Kenya. $\mathrm{Br}$ J Nutr. 2007;98:422-30.

10. Schroten H, Bosch M, Nobis-Bosch R, Köhler H, Hanisch FG, Plogmann R. Secretory immunoglobulin A is a component of the human milk fat globule membrane. Pediatr Res. 1999;45:82-6.

11. Semba RD. The role of vitamin $A$ and related retinoids in immune function. Nutr Rev. 1998;56:S38-48.

12. Duriancik DM, Lackey DE, Hoag KA. Vitamin A as a regulator of antigen presenting cells. J Nutr. 2010;140:1395-9.

13. Maruya M, Suzuki K, Fujimoto H, Miyajima M, Kanagawa O, Wakayama T, et al. Vitamin A-dependent transcriptional activation of the nuclear factor of activated T cells c1 (NFATc1) is critical for the development and survival of B1 cells. Proc Natl Acad Sci U S A. 2011;108:722-7.

14. Ballabio C, Bertino E, Coscia A, Fabris C, Fuggetta D, Molfino $S$, et al. Immunoglobulin-A profile in breast milk from mothers delivering full term and preterm infants. Int J Immunopathol Pharmacol. 2007;20:119-28.

15. Weaver LT, Austin S, Cole TJ. Small intestinal length: a factor essential for gut adaptation. Gut. 1991;32:1321-3.

\section{Correspondence:}

Roberto Dimenstein

Departamento de Bioquímica, Centro de Biociências

Universidade Federal do Rio Grande do Norte (UFRN)

Av. Senador Salgado Filho, 3000, Lagoa Nova

CEP 59072-970 - Natal, RN - Brazil

Tel.: + 55 (84) 3215.3416, ext. 205

Fax: +55 (84) 3211.9208

E-mail: rdimenstein@gmail.com 